\title{
Außer Konkurrenz? Strategien der Inanspruch- nahme einer Südtiroler Landarztpraxis um 1890 bei Erkrankungen von Kindern
}

Alois Unterkircher

\section{Summary}

Who was responsible for the treatment of sick children in the countryside during the second half of the 19th century? This paper investigates the medical complaint accusing the rural population of only reluctantly bringing their sick offspring to academic physicians. The following analyses the role Franz v. Ottenthal (1818-1899), a 'representative' of a private rural medical practice, played in the specialised medical market attending to childhood diseases. An exemplary survey of Ottenthal's medical records for patients from the age of one to 14 years throughout the 1890s has shown that children contributed a relevant percentage of the whole of the physician's patient distribution. It may therefore be assumed that Ottenthal knew how to successfully merchandise his specific therapies. On the demand side, however, parents of sick children were not solely reliant upon this physician. Evidence from the medical records provides information as to when parents regarded medical self-help as no longer supporting the recovery of their children, the cures of lay healers failed, or cases when parents were not satisfied with the therapeutic treatments other physicians had to offer and therefore consulted Ottenthal.

Keywords: child patients, child health, rural medical practice, medical market, 19th century, Tyrol

\footnotetext{
* Dieser Beitrag enthält Ergebnisse des Dissertationsprojektes «Männer als Patienten. Krankheitsverhalten von Männern im ländlichen Raum in der zweiten Hälfte des 19. Jahrhunderts am Beispiel des Südtiroler Landarztes Franz von Ottenthal», das von der Robert Bosch Stiftung Stuttgart gefördert wurde, sowie erste Forschungen aus dem Projekt «Praxis und PatientInnen des Südtiroler Landarztes Franz von Ottenthal (1818-1899)», gefördert durch die Autonome Provinz Bozen - Südtirol, Abteilung Bildungsförderung, Universität und Forschung.
}

Dr. Alois Unterkircher, Institut für Geschichtswissenschaften und Europäische Ethnologie, Universität Innsbruck, Innrain 52, A-6020 Innsbruck (alois.unterkircher@uibk.ac.at). 


\section{Zusammenfassung}

Wer war in der zweiten Hälfte des 19. Jahrhunderts am Land für die Behandlung kranker Kinder zuständig? Dieser Beitrag geht von den vielerorts vorgebrachten ärztlichen Klagen aus, die Landbevölkerung hätte ihren Nachwuchs im Erkrankungsfall nur selten in die Praxen akademischer Ärzte gebracht. Im Folgenden wird daher die Rolle, die Franz v. Ottenthal (1818-1899) als 〈Vertreter〉 einer privaten Landarztpraxis am medizinischen Markt für Kinderkrankheiten eingenommen hatte, auf Basis einer exemplarischen Analyse seiner Praxisjournale aus den 1890er Jahren herausgearbeitet. Eine Auswertung der entsprechenden Krankengeschichten (Kinder von 1 bis 14 Jahren) zeigte, dass Kinder einen anteilsmäßig relevanten Prozentsatz am gesamten Patientenstock dieses Arztes einnahmen. Ottenthal wusste seine spezifischen Therapien demnach mit Erfolg zu vermarkten. Nachfrageseitig stellte für die Eltern kranker Kinder die Wahl dieses Arztes allerdings nicht die einzige Option dar: Hinweise in den Krankenjournalen geben Auskunft darüber, wann in den Augen der Eltern medizinische Selbsthilfe die Genesung ihrer Kinder nicht mehr unterstützte, wann die Mittel von Laienheilern versagten oder wann Eltern mit den Therapieangeboten ärztlicher Fachkollegen nicht zufrieden waren und zu Ottenthal wechselten.

Schlagworte: Kinder als Patienten, Gesundheit im Kindesalter, ärztlicheVersorgung auf dem Land, medizinischer Markt, 19. Jahrhundert, Tirol

Im Dezember 1860 schickte der in der Kleinstadt Bruneck ansässige Bezirksarzt seinen Bericht über eine zu diesem Zeitpunkt hauptsächlich unter den Kindern grassierende Masernepidemie in Enneberg an die zuständigen Behörden. Für den Sanitätsbeamten waren die Schuldigen an den hohen Opferzahlen dieser Epidemie schnell ausfindig gemacht, denn: «Die kranken Kinder werden meistens mit Hausmittel, als Eibisch- und Himmelbrandthee, warmes Verhalten, und warme Umschläge auf den Bauch etc. behandelt und die wenigsten Eltern fühlen sich gedrungen für ihre kranken Kinder ärztliche Hilfe in Anspruch zu nehmen.» ${ }^{1}$ Für den Bezirksarzt wären offensichtlich viele Kinder dem Tod entronnen, hätten deren Eltern nicht aus Teilnahmslosigkeit und Starrsinn auf den kompetenten Rat eines Arztes verzichtet. Die anscheinende Sorglosigkeit vieler Eltern, Krankheitsverläufe ihrer Kinder allein mit einfachen Hausmitteln zu einem guten Ende bringen zu wollen,

1 TLA, Akten Statthalterei 1860, Sanität; Zl. 35250/2714, ad 1923 Sanität. «Bericht des k.k. Bezirksarztes zu Bruneck über den Ausbruch des Masern und der Ruhr unter den Kindern zu St. Vigil in Enneberg vom 24.12.1859.» 
beklagte ein weiterer Arzt nur wenig später. Anlässlich einer in einem Dorfe nahe Innsbruck grassierenden Masernepidemie meldete dieser 1866: «Ärztliche Hilfe wurde nur spärlich angesprochen; sobald die Leute wussten, dass es sich um das erwähnte Exanthem handle, wurde nur zu den gewöhnlichen Hausmitteln, besonders schweißtreibenden Thees gegriffen.» ${ }^{2}$

Nun haben sich derartige Klagen von Ärzten über die fehlende Einsicht von Eltern, für ihren erkrankten Nachwuchs einen Arzt in Anspruch zu nehmen, in den Archiven zu Dutzenden erhalten. Bei genauerem Hinsehen erscheinen die Motive der Eltern für oder gegen eine ärztliche Konsultation jedoch in einem etwas anderen Licht. So erfahren wir an anderer Stelle des ersten Berichtes über die Masernepidemie, dass die einzelnen Krankheitsverläufe zu Beginn äußerst milde verlaufen wären, ehe sie unvermutet einen «bösartigen Character» entwickelt und mehrere Kinder innerhalb kürzester Zeit dahingerafft hätten. Es war demnach die anfängliche Einschätzung einer gewöhnlichen und ohne Komplikationen verlaufenden Masernerkrankung, die viele Eltern zuerst zu Hausmitteln greifen und sie erst später - in den Augen des Bezirksarztes zu spät - einen akademischen Arzt in Anspruch nehmen ließ. Zu diesem Zeitpunkt hatte sich die Krankheit vielerorts schon zu einer ernsthaften Bedrohung für das Leben der betroffenen Kinder ausgewachsen. Auch der zweite Bericht spricht davon, dass das elterliche Abwägen zwischen medizinischer Selbsthilfe oder ärztlicher Zuhilfenahme eigentlich erst entschieden wurde, als die beobachteten Symptome einem «alltäglichen» Krankheitsbild zugeordnet werden konnten - nämlich den häufig erlebten und vermutlich auch selbst durchlebten Masern.

Von einer grundsätzlichen Ablehnung der gelehrten Medizin und einem allgemeinen Misstrauen gegenüber Ärzten kann folglich keine Rede sein, ebenso wenig von einer vor dem Hintergrund hoher Kindersterblichkeit fatalistischen Gleichgültigkeit gegenüber kranken Kindern oder dem Kindstod. ${ }^{3}$ Vielmehr scheint der Entscheidungsfindungsprozess zur HeilerInnenwahl wohl einer eigenen Logik gefolgt zu sein, bei der die Bedrohlichkeit einer Erkrankung, die Möglichkeiten der medizinischen Selbsthilfe und das Hinzuziehen eines Arztes sehr überlegt gegeneinander abgewogen wurden. Vor dem Hintergrund der um 1860 insgesamt noch recht beschränkten Heilerfolge der Medizin erscheint die Klage der Ärzte somit in erster Linie als ein Lamentieren über die mangelnde Nachfrage nach spezifisch ärztlichen Therapieangeboten, die von den Eltern zugunsten anderer Möglichkeiten kurativer Einflussnahme links liegen gelassen wurden.

2 TLA, Akten Statthalterei 1866, Sanität; Zl. 336/23. «Bericht des k.k. Kreisarztes zu Innsbruck vom 12. Jänner 1866 über die Masern in Amras.»

3 Zu diesem Aspekt vgl. die differenzierten Aussagen bei Wolff 1995, 127-129. 
In Hinblick auf das Thema dieses Bandes ergeben sich aus diesen knappen Ausführungen folgende Fragen: Inwiefern waren die medizinischen Dienstleistungen, die Franz v. Ottenthal (1818-1899) als praktischer Arzt in einem Südtiroler Gebirgstal im Falle einer Erkrankung von Kindern anzubieten hatte, für Eltern attraktiv und somit tatsächlich «außer Konkurrenz»? In welchen Bereichen der Kindermedizin musste sich Ottenthal seinen MitanbieterInnen und KonkurrentInnen geschlagen geben, die die elterliche Nachfrage nach einer Versorgung ihres erkrankten Nachwuchses geschickter zu bedienen wussten? Und mit wem teilte sich Ottenthal dieses spezielle Segment am medizinischen Markt überhaupt, wo doch die Bevölkerung Ende des 19. Jahrhunderts angesichts der Entwicklungen und Spezialisierungen in der Kinderheilkunde doch längst schon zu «medikalisierten PatientInnen» erzogen hätte sein müssen ${ }^{4}$ und den ärztlichen «Expertenstatus» bei kindermedizinischen Fragen gar nicht mehr in Frage hätte stellen dürfen. ${ }^{5}$

Die von Elena Taddei verfasste Biographie Franz v. Ottenthals liegt seit 2010 publiziert vor, so dass an dieser Stelle lediglich die wichtigsten Eckdaten im Leben dieses Arztes zusammengefasst werden müssen. ${ }^{6}$ Geboren am 23. Mai 1818 als ältestes von drei Kindern einer Familie aus dem ländlichen Tiroler Kleinadel, begann Ottenthal im Herbst 1837 mit einem Studium an der Medizinischen Fakultät in Wien. Sein Diplom als Doktor der Medizin erhielt er im Juni 1843, jenes als Doktor der Chirurgie im Oktober desselben Jahres. Im Februar 1844 legte er zusätzlich ein Rigorosum über Geburtshilfe ab. Zwischen 1844 und 1846 arbeitete er zunächst als Gemeindearzt im Bezirksgericht Windisch-Matrei (heute Matrei in Osttirol), ehe er den Sprung in die Selbständigkeit wagte. Im Jänner 1847 eröffnete er seine Privatpraxis in der zu dieser Zeit rund 740 Seelen zählenden Gemeinde Sand, dem Verwaltungssitz des Bezirksgerichts Taufers (ca. 9000 EinwohnerInnen). ${ }^{7}$ Ottenthal führte seine Landarztpraxis ohne Unterbrechung bis zum 29. Jänner 1899, als er hochbetagt und nach rund 52 Jahren ärztlicher Tätigkeit im Bezirk Taufers verstarb.

4 Zum Konzept der Medikalisierung vgl. Loetz 1993.

5 Von der traditionellen Medizingeschichtsschreibung wurden Kleinkinder und Kinder nur selten als eigenständige Patientengruppe problematisiert. Ausnahmen sind etwa die Beiträge von Ritzmann 2003, 163-178, Ruisinger 2005, 218-236, und Ritzmann 1999, 189-208, zu ausgewählten Aspekten der Kinderheilkunde. Zuletzt füllte Ritzmann mit ihrer Monographie diese Lücke, allerdings nur für das 18. Jahrhundert. Vgl. Ritzmann 2008.

6 Vgl. Taddei 2010.

7 Im Zuge der Angliederung Südtirols an den italienischen Staat wurde die alte «k. u. k. Struktur» der Bezirksämter aufgelöst. Das ehemalige «Bezirksamt Taufers〉 besteht heute aus den fünf Großgemeinden Gais, Sand, Mühlwald, Ahrntal und Prettau. Vgl. dazu Innerhofer 1980, $320 \mathrm{f}$. 


\section{Kinder als Patienten Franz v. Ottenthals}

Vor der Erörterung der angesprochenen Fragen soll zunächst geklärt werden, ob sich das in unzähligen Epidemieberichten und ärztlichen Klagen vermittelte Bild der geringen Nachfrage nach einer Behandlung kranker Kinder durch akademische Ärzte überhaupt mit der täglichen Routine in einer Praxis deckt. Falls ja, müsste der Anteil jüngerer Altersgruppen an der gesamten Patientenschaft Franz v. Ottenthals verschwindend gering sein. Dies ist aber überhaupt nicht der Fall. Exemplarisch für das Jahrzehnt der 1890er Jahre berechnet waren Kleinkinder mit rund fünf Prozent und Kinder im Alter zwischen vier und vierzehn Jahren mit ca. neun Prozent in der Praxis Ottenthals vertreten (vgl. Grafik 1). Zählt man die Altersgruppe der Säuglinge hinzu, so hatte in diesem Jahrzehnt ein gutes Fünftel aller PatientInnen Ottenthals das Jugendalter noch nicht erreicht. Auch eine Detailanalyse der einzelnen Tage des Monats März, verteilt auf ausgewählte Jahre der letzten Dekade des 19. Jahrhunderts, ergab nur wenige Tage, an denen Ottenthal nicht mindestens einmal um Hilfe für ein erkranktes Kind nachgefragt wurde. Von den 31 potenziellen Besuchstagen des März blieben nur 12 «kinderfrei». Entgegen den ärztlichen Klagen gehörte folglich der Anblick von Kindern - oder zumindest von deren in einem Fläschchen gebrachten Urin ${ }^{8}$ - durchaus zum Alltag in einer ärztlichen Praxis.

Mit einem ermittelten Anteil von 14 Prozent muss die Praxis Ottenthals in dieser Hinsicht als durchschnittliche Arztpraxis gesehen werden, denn Martin Dinges errechnete für verschiedene Arztpraxen des 17. bis 20. Jahrhunderts einen Kinderanteil zwischen sieben und 14 Prozent mit Maximalwerten in beide Richtungen. ${ }^{9}$ Dass der Befund zur Ottenthal'schen Praxis kein regionales Spezifikum des Tauferer Ahrntals darstellt, belegt nicht zuletzt die zeitlich und geographisch zu dieser Arztpraxis sehr nahe liegende Studie zu Niederbayern von Michael Stolberg. Seine Auswertungen von neun ärztlichen Physikatsberichten aus den frühen 1860er Jahren ergaben hinsichtlich einer altersspezifischen Aufschlüsselung der Patientenschaft annähernd gleiche Werte wie für das Südtiroler Quellenmaterial. Die Altersgruppe zwischen zwei und zehn Jahren stellte in den niederbayerischen Praxen neun bis zwölf Prozent, jene der Elf- bis Zwanzigjährigen rund neun Prozent der PatientInnen. ${ }^{10}$

8 So heißt es im Bericht aus dem Jahre 1866 weiter: «In schlimmen Fällen, wurde der Urin zum Arzte geschickt und auf gut Glück ordiniert, so bei den 2 verstorbenen Kindern.» Vgl. Anm. 2. Auf das Problem möglicher Botendienste und Briefkonsultationen kann in diesem Beitrag allerdings nicht näher eingegangen werden.

9 Dinges 2007, 303f., Tab. 3.

10 Stolberg 1993, $19 f$. 


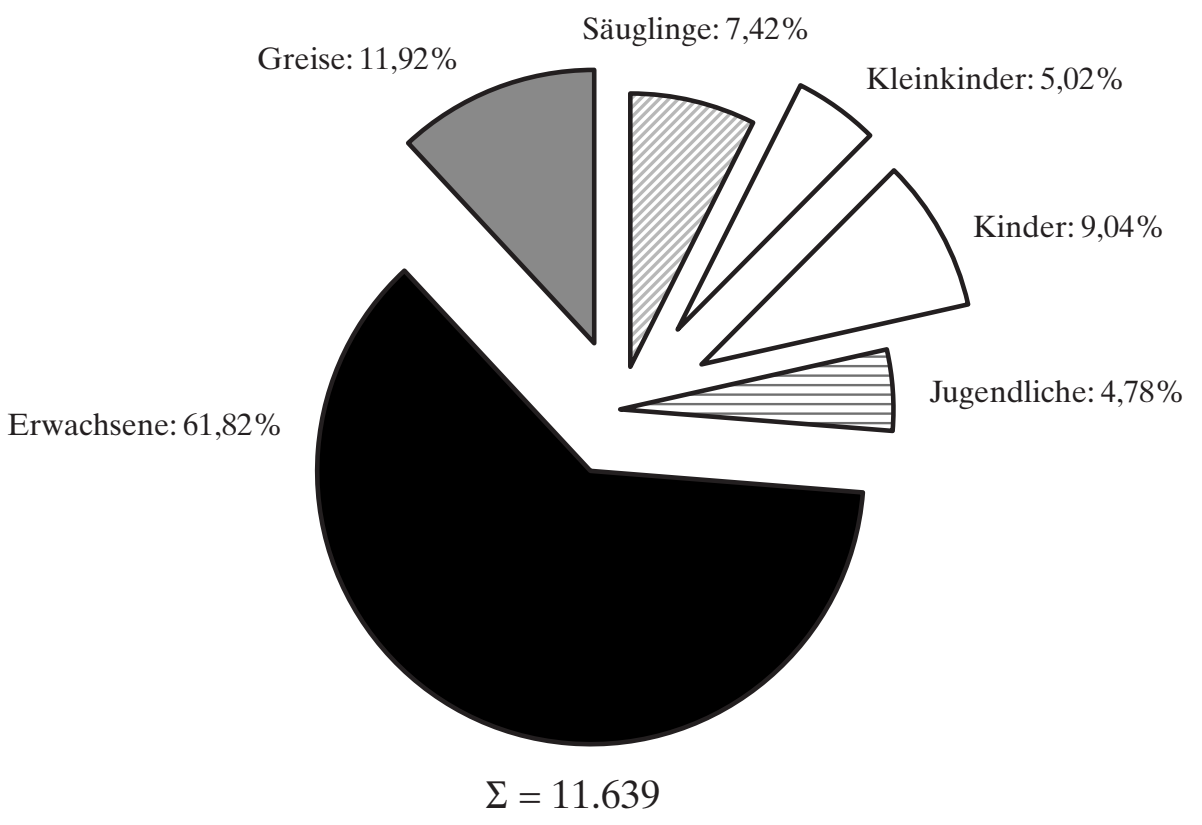

Grafik 1:Prozentualer Anteil der Kleinkinder (1-4 Jahre) und der Kinder (4-14 Jahre) an der gesamten Patientenschaft Ottenthals im Jahrzehnt 1890-1899.

Quelle: SLA, Nachlass Ottenthal, Historiae Morborum 1890-1899.

Nun könnte man einwerfen, dass diese 14 Prozent kindlicher PatientInnen lediglich aus den Dörfern der unmittelbaren Nachbarschaft stammten. Die Eltern hätten somit weder lange Distanzen in die Praxis überwinden noch im Falle einer Visite das teure Weggeld aufbringen müssen. Den Eltern wurde wiederum der Segen eines raschen ärztlichen Eingreifens in den Krankheitsverlauf sichtbar vor Augen geführt, beispielsweise durch die Tracheotomie, die bei den Erstickungsanfällen in Folge schwerer Diphtherieerkrankungen mitunter lebensrettend sein konnte. ${ }^{11}$ Doch auch das Argument der räumlichen Distanz als Faktor für die ärztliche Inanspruchnahme überzeugt nicht völlig, denn in den 1890er Jahren setzten sich die kindlichen PatientInnen Ottenthals nur zu rund 66 Prozent aus der Bevölkerung des näheren Einzugsgebietes des Arztes zusammen (vgl. Grafik 2). Rund 27 Prozent aller Kinder hatten ihren Wohnsitz in einer der fünf Gemeinden des Ahrntals, die über einen eigenen Gemeindearzt in Steinhaus verfügten. Ein geringer Teil (3,7\%) kam aus der näheren Umgebung von Bruneck und den Nachbarbezirken, wo ebenfalls andere Ärzte schneller zu erreichen gewesen wären. Hingegen stammten mit einem Geschwisterpaar nur zwei aller 1636 behandelten Kinder aus einem anderen

$11 \mathrm{Zu}$ diesem Verfahren und zur Entwicklung der Diphtheriesterblichkeit in Österreich in den 1890er Jahren vgl. Pammer 2005, 79. 


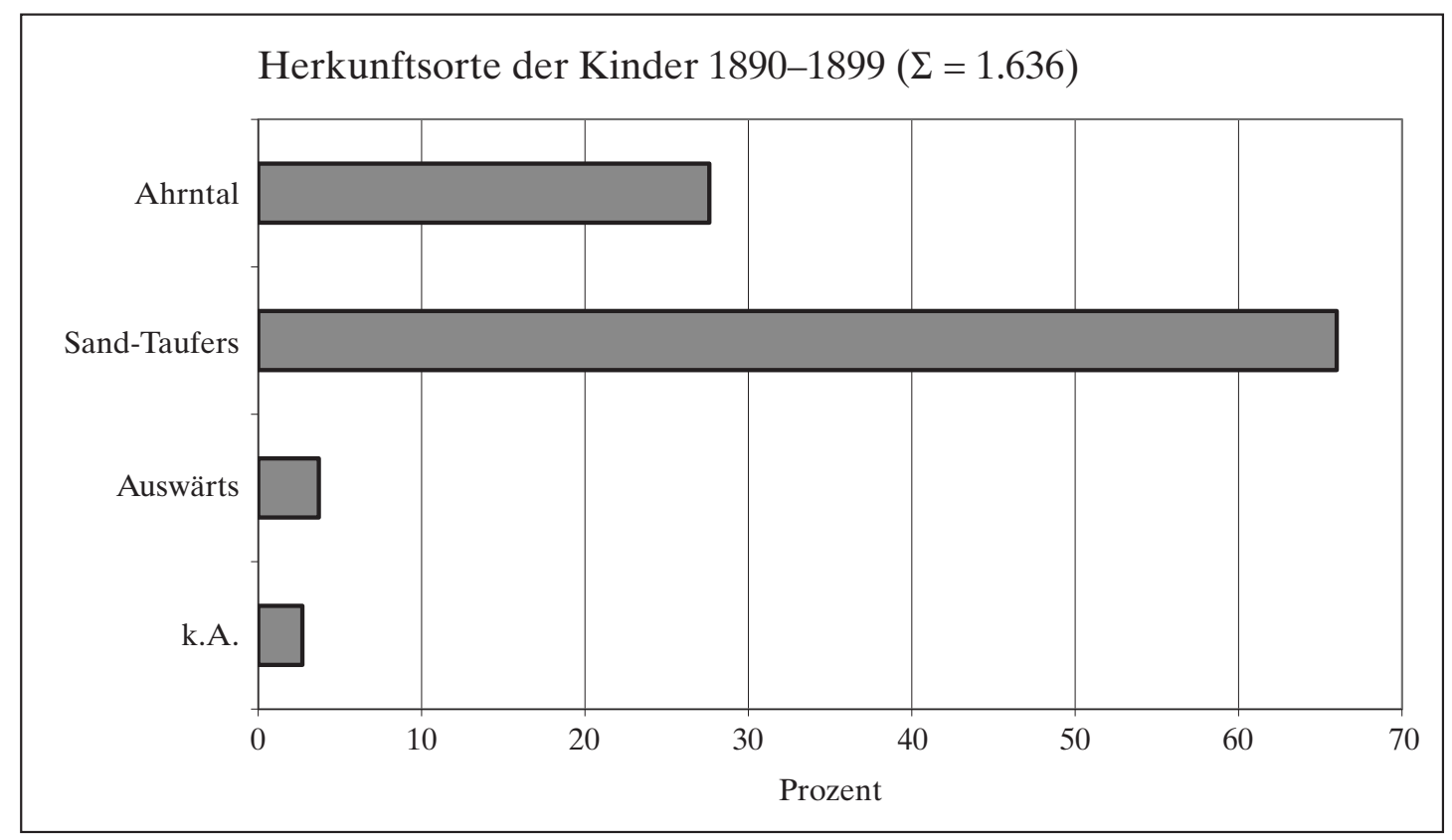

Grafik 2: Herkunftsort der Kinder 1890-1899.

Quelle: SLA, Nachlass Ottenthal, Historiae Morborum 1890-1899.

Bezirk Tirols (Flaurling im Tiroler Oberland), wobei es sich in diesem Fall um in das Tauferer Ahrntal vermittelte Pflegekinder gehandelt haben dürfte.

Diese Befunde belegen eindrücklich, dass Eltern sehr wohl die Hilfe von akademischen Ärzten in Anspruch nahmen, wenn sie sich dadurch eine Besserung des Krankheitszustandes ihrer Kinder erhofften. Bei der Behandlung der jüngeren Patientenschaft wurden Ottenthal und seine Landarztpraxis allerdings hauptsächlich von der lokalen Bevölkerung nachgefragt, er vermochte es nicht, Eltern mit ihren erkrankten Kindern aus weiter entfernten Regionen anzuziehen. Wie ein Vergleich mit den Journalen der 1860er Jahre zeigt, hat sich der Anteil der Kinder an der gesamten Patientenschaft nur geringfügig verändert (Kleinkinder 4,3\%, Kinder 7,7\%). PatientInnen in den jüngeren Altersklassen waren für Ottenthal demnach trotz einer sich im Verlauf des 19. Jahrhunderts zunehmend ausdifferenzierenden Kindermedizin kein lukrativer «Wachstumsmarkt». ${ }^{12}$ Doch welche

12 Die bisherigen Ergebnisse zu Kindern als Patienten Ottenthals sprechen gegen die These, die Durchführung der (Schutz-)Pockenimpfung könnte für diesen stattlichen Kinderanteil in der Arztpraxis mit verantwortlich gewesen sein. Die Historiae Morborum enthalten zwar einige Hinweise zur Impfung. Die knappen Notizen Ottenthals zu dieser medizinischen Technik belegen allerdings nur, dass er einige durch die Impfung verursachte «Folgeschäden» behandeln musste, und nicht, dass er die Vakzination auch selbst durchgeführt hat. So heißt es etwa bei einem geimpften Säugling: $« 8$ mens. heri vaccinata refert. in circumferentia inflammationem et laborat convulsionibus». HM 738/1871, Eintrag vom 29.5.1871. Eine «offizielle» Bestellung zum Impfarzt ist nur für bestimmte Jahre (etwa 1884) überliefert, als er provisorisch Aufgaben des Gemeindearztes übernommen hatte. Siehe Taddei 2010, 166-168. 
Heilkundigen außer Ottenthal hätten die Eltern um 1890 in der Region um Hilfe für ihre kranken Kinder aufsuchen können? Wie sah der regionale medizinische Markt aus?

\section{Der medizinische Markt im Bezirk Taufers um 1890}

Der politische Bezirk Bruneck umfasste im Untersuchungszeitraum die vier Bezirkshauptmannschaften Bruneck, Enneberg, Welsberg und Taufers mit zusammen rund 34919 EinwohnerInnen, wovon rund 9000 auf den Bezirk Taufers entfielen. ${ }^{13} \mathrm{Zu}$ dieser Zeit war die Organisation des Gesundheitswesens auf regionaler Ebene durch die nach langwierigen Verhandlungen im Jahre 1884 endgültig eingerichteten Sanitätssprengel schon sehr gut strukturiert. In jedem Sprengel des Verwaltungsgebietes Tirol/Vorarlberg musste ein Gemeindearzt oder -wundarzt für die Bevölkerung verfügbar sein. Der Bezirk Taufers war dabei in zwei Sanitätssprengel (Sand-Taufers mit zehn Gemeinden und Ahrntal mit fünf Gemeinden) aufgeteilt. ${ }^{14}$ Auffallend ist die im Vergleich zu anderen Regionen geringe Ärztedichte in diesem Bezirk: Laut einer Statistik musste um 1880 in Tirol ein Arzt im Durchschnitt 1633 Personen versorgen, wohingegen im Bezirk Bruneck ein Arzt 2352 Personen medizinisch zu betreuen hatte. ${ }^{15}$ Mit diesem Betreuungsschlüssel lag der Bezirk von sämtlichen 25 Verwaltungsbezirken Tirols an viertletzter Stelle, nur die Distrikte Primiero, Ampezzo und Trient-Umgebung mussten im Verhältnis zu ihren Bevölkerungen mit weniger Ärzten auskommen.

Noch dazu ließ sich die überwiegende Mehrzahl der Ärzte dieses Bezirkes in Gemeinden entlang der Hauptverkehrsstraße durch das Pustertal nieder, wohingegen die schwer zugänglichen Gebirgstäler mit ihren oftmals zerstreuten Siedlungen verhältnismäßig schlecht von akademischen Ärzten versorgt wurden. Noch in einem Verzeichnis der Gemeindeärzte aus dem Jahre 1909 wurden sowohl der Sprengel Sand-Taufers als auch jener von Ahrntal als «ausgedehnt und beschwerlich» bezeichnet, ${ }^{16}$ und Franz v. Ottenthal selbst gibt einen Einblick in die Strapazen, die eine regelmäßige Bereisung und eine akute Notversorgung für einen dort niedergelassenen Arzt bedeuteten. Auf die Verwarnung der zuständigen Bezirksbehörden Anfang 1895 hin, warum Ottenthal den Ausbruch der Diphtherie bei eini-

13 K.K. Statistische Central-Commission 1893, 50.

14 Vgl. TLA, Akten Statthalterei 1890, Sanität; Nr. 2078 ad Zl. 5199/565, liegend in Zl. 2615. «Bericht des k.k. Bezirkshauptmannes vom 1.3.1890 an die k.k. Statthalterei.» Zu Tirol allgemein vgl. N.N. 1897, 482-485.

15 K.K. Statistische Central-Commission 1882, XXIIIf.

16 Sanitätssprengel 1909, 11-13. 
gen Kindern eines entlegenen Hofes im Dorf Mühlwald nicht wie sanitätspolitisch vorgeschrieben gemeldet hatte, rechtfertigte sich der Arzt folgendermaßen: «[...] der hoch auf dem Berg gelegene E.hof in Mühlwald ist $1 \frac{1}{2}$ bis 3 Gehstunden von Sand entfernt, der Gang dahin - gefahren kann nicht werden - wäre für einen 77jährigen Greis bei dem heurigen Winter und den schlechten Wegverhältnissen mit augenscheinlicher Erkrankungsgefahr verbunden.» ${ }^{17}$

Die im letzten Drittel des 19. Jahrhunderts vielerorts vorgebrachte Klage über den Ärztemangel in Tirol betraf demnach weniger bevölkerungsreiche Gemeinden mit zentralörtlicher Funktion als vielmehr gebirgige und abseits der Durchzugsstraßen gelegene Hochgebirgstäler wie das hintere Ahrntal. ${ }^{18}$ In der dortigen Gemeinde Steinhaus befand sich über Jahrhunderte hinweg ein Wundarzt, der v.a. die durch Arbeitsunfälle bedingten Verletzungen der Bergleute des Prettauer Kupferbergwerks chirurgisch zu versorgen hatte. Dessen Wartgeld teilten sich die dortigen Gemeinden und das in Privatbesitz befindliche Bergbauunternehmen zu gleichen Teilen. ${ }^{19}$ Spätestens mit der Schließung des wirtschaftlich nicht mehr konkurrenzfähigen Bergbaubetriebes 1893 fiel dieser fixe Patientenstock weg, und für die Gemeinden selbst wurde es zusehends schwieriger, einen Wundarzt in diese abgelegene Region zu locken. ${ }^{20}$ Als beispielsweise auf die im Jänner 1891 ausgeschriebene Gemeindearztstelle in Steinhaus keine geeigneten Bewerbungen eingegangen worden waren, hoben die fünf Gemeinden das Wartgeld bei der neuerlichen Ausschreibung im April desselben Jahres von 400 auf 450 Gulden an. ${ }^{21}$ Ungeachtet derartiger finanzieller Anreize hatte die Wundarztstelle in dieser für Ärzte offenbar unattraktiven Gegend eine hohe Fluktuation aufzuweisen und blieb die gesamten 1890er Jahre mehrmals unbesetzt.

Trotz einer im Vergleich zu anderen Regionen äußerst geringen Anzahl an akademisch geschultem Heilpersonal darf nicht davon ausgegangen werden, die Eltern erkrankter Kinder hätten um 1890 nur zwischen den wenigen akademischen Therapeuten oder der medizinischen Selbsthilfe wählen können. Vielmehr stand der Bevölkerung noch eine Vielzahl anderer Möglichkeiten offen, um ihren Kindern die ihrer Einschätzung nach bestmögliche Therapie zu beschaffen. So gab es neben Franz v. Ottenthal als praktischem Arzt den Gemeindearzt in Sand und jenen in Steinhaus. Die

17 Zit. nach Taddei 2010, 163.

18 Vgl. dazu Dietrich-Daum 2008, 210-221.

19 Messner 2004, $182 \mathrm{f}$.

20 Von diesem «Ärztemangel» waren auch andere Regionen betroffen. Vgl. Taddei 2008, 232-235.

21 Siehe die Ausschreibungen im Beiblatt zur Wochenschrift «Das österreichische Sanitätswesen» 1891,17 und 69. 
Bevölkerung des Tauferer Talbodens konnte ihre kranken Kinder zudem vom Stadtarzt in Bruneck, vom Wundarzt in Bruneck oder vom Gemeindearzt des unweit östlich dieser Bezirksstadt liegenden Dorfes St. Lorenzen behandeln lassen. Außerdem waren in zwölf Gemeinden des Tales Hebammen angestellt. ${ }^{22}$ Vielleicht nahm der eine oder die andere TalbewohnerIn aber auch beim ohnehin erforderlichen Gang in die einzige Apotheke des Bezirks in Bruneck noch ein Mittel für ein erkranktes Kind mit, oder der ebenfalls in der Stadt ansässige Bezirkstierarzt steckte neben einem Mittel gegen den «Kuhdiesel» gleich stillschweigend ein mildes Laxans für die Kinder eines Bauern mit in Tasche. Für die Behandlung schwerwiegenderer Verletzungen oder ansteckender Krankheiten, die wie die Syphilis einer längeren Kur bedurften, standen mit dem Stadtspital in Bruneck und dem Versorgungshaus in Mühlen (unweit Sand), welches von den Barmherzigen Schwestern besorgt wurde, entsprechende Institutionen zur Verfügung. Daneben boten zahlreiche männliche und weibliche Laienheiler therapeutische Hilfe an, allerdings sind diese quellenmäßig nur schwer und für die neuere Zeit hauptsächlich über die volkskundliche Regionalforschung zu fassen. Der in Steinhaus zu Beginn des 20. Jahrhunderts wirkende Alois Hofer beispielsweise galt als Spezialist bei Knochenbrüchen ${ }^{23}$ und im Fall des Johann Voppichler*24 aus St. Johann wurde 1867 anlässlich einer Konsultation von Ottenthal sogar die Bezeichnung «agyrta» als Beruf notiert. ${ }^{25}$ Letztlich stand selbstverständlich auch eine ganze Armee von nahen und entfernten Verwandten, Nachbarn, Freunden und Bekannten sofort parat, um die besorgten Eltern mit eigenen Erfahrungsberichten über kompetente Heiler und zuverlässig wirkende Heilmittel zu versorgen. Dabei erregte hauptsächlich der weibliche Teil dieser sozialen Netzwerke in Gestalt von Großmüttern, Tanten oder Ammen den Zorn der Ärzte. «Alte Kinderfrauen erfreuen sich bei Ärzten keiner Beliebtheit, weil sie als unbelehrbar gelten», ${ }^{26}$ fasst der Berliner Kinderarzt Adalbert Czerny (1863-1941) die Einschätzungen vieler seiner Fachkollegen um 1900 zusammen.

Franz v. Ottenthal führte seine Landarztpraxis also keinesfalls «außer Konkurrenz», sondern teilte sich den medizinischen Markt mit vielen anderen AnbieterInnen. Daher soll im Folgenden untersucht werden, inwieweit

22 TLA, Akten Statthalterei 1896, Sanität; Zl. 35.955/2772, liegend in 3541. «Verzeichnis der im politischen Bezirke Bruneck a, 1. December 1896 vorhandenen Hebammen».

23 Vgl. Egger 1999, 60-69, hier 65.

24 Aus Datenschutzgründen handelt es sich bei einer mit * gekennzeichneten Person um ein Pseudonym.

25 Historiae Morborum [fortan HM] 892/1867, Eintrag vom 5.6.1867. In Fachlexika wird das griechische Wort «Agyrtes» mit «Marktschreier» übersetzt. Papes Handwörterbuch ${ }^{3} 1908$, 25.

26 Czerny ${ }^{8} 1934,22$. 
sich aus den Praxisjournalen Ottenthals mögliche Motive herausfiltern lassen, wann Eltern erkrankter Kinder neben anderen medizinischen Kuren schlussendlich seine Privatpraxis in Anspruch nahmen. Ist eine ähnliche «Stufengliederung der Therapeuten ${ }^{27}$ mit kinderheilkundigem Angebot, wie sie Iris Ritzmann anhand von Quellen aus dem 18. Jahrhundert aufgestellt hat, festzustellen?

\section{Selbstmedikation und medizinische Selbsthilfe}

Für gewöhnlich versuchten Eltern bei ersten Anzeichen von Unwohlsein und Krankheiten ihrer Kinder, wenn möglich in den eigenen vier Wänden eine angemessene Versorgung und Pflege der Betroffenen zu gewährleisten. ${ }^{28}$ Die aufgetretenen Beschwerden wurden nach ihrer Gefährlichkeit beurteilt, erprobte Hausmittel eingesetzt, der begehrte Platz in der Nähe des wärmenden Ofens eingerichtet. Die Einholung eines ärztlichen Rats war nur eine Option unter mehreren.

Das Beispiel des eineinhalbjährigen Simon Auer* aus Lappach kann als charakteristisch für einen solchen Prozess der elterlichen Entscheidungsfindung gelten. Franz v. Ottenthal notierte am 11. Februar 1891 in sein Journal: «1 $1 \frac{1}{2}$ anni ex 6 septimanis laborat diarrhoea, quae remediis domesticis non cedit». ${ }^{29}$ Demnach versuchten die Eltern über einen längeren Zeitraum hindurch, den Durchfall ihres Sohnes mit verschiedensten Hausmitteln zum Stillstand zu bringen. Erst als die Wirkungslosigkeit ihrer eigenen Rezepturen offensichtlich wurde, suchten sie Hilfe bei Ottenthal. Seine Medikation führte zunächst eine Besserung herbei, denn erst im darauffolgenden Monat fand eine zweite Konsultation statt. Ottenthal merkte im Zuge seiner Niederschrift allerdings an, dass es nur zu einer vorübergehenden Zurückdrängung des Durchfalls gekommen war, woraufhin er mit einem aus anderen Bestandteilen zusammengesetzten Rezept sein neuerliches Glück versuchte. Auch die Eltern der eineinhalbjährigen Maria Zimmerhofer* aus Ahornach entschlossen sich nach fünf Wochen vergeblichen Bemühens, den Durchfall mit erprobten Mitteln zu bekämpfen, schließlich doch zur Einholung eines ärztlichen Rats. ${ }^{30}$ Freilich berichten die Krankenjournale nicht nur von der Wirkungslosigkeit derartiger Versuche medizinischer Selbsthilfe. «[A]nte 3 dies noctu adficiebatur enteralgia remediis domesticis cedente»,

27 Ritzmann 2003, $172 \mathrm{f}$.

28 Ritzmann 2008, 87-91.

29 HM 229/1891, Eintrag vom 11.2.1891.

$30 \ll 1 \frac{1}{2}$ anni ex 5 septimanis laborat diarrhoea remediis non cedente domesticis, hinc inde vomituritio, magnus appetitus, lingua pura.» HM 1492/1895, Eintrag vom 10.10.1895. 
klärt Ottenthal über den Krankheitsverlauf des neunjährigen Johann* auf. ${ }^{31}$ Letztendlich gaben jedoch nicht die Darmschmerzen als vielmehr das unvermutet einsetzende Kopfweh, der Schmerz hinter dem rechten Ohr und die fortwährenden Delirien mit Temperaturwechseln den entscheidenden Impuls für die Eltern, beim Arzt um Hilfe anzusuchen.

Einige der Hausmittel werden in den Quellen namentlich genannt, so etwa Kamillentee, ${ }^{32}$ Kümmelöl, ${ }^{33}$ zwei Tropfen Baldrian ${ }^{34}$ oder die Jalapenwurzel, ${ }^{35}$ welche der fünfjährige Jakob Kammerlander* 1891 als in Wein aufgelöstes Pulver gegen seine Bauchschmerzen und seine Appetitlosigkeit zu trinken bekam. Als ein um wenige Jahre jüngerer Knabe aus Luttach von Fieber und Erbrechen befallen wurde, versuchten die Eltern zunächst mit Mannasirup eine Linderung zu erreichen. ${ }^{36}$ Manna galt gerade bei hitzigen und entzündlichen Fiebern als mild wirkendes Purgiermittel mit geringen Nebenwirkungen und somit als äußerst verträglich für den in der Pädiatrie bis weit ins 19. Jahrhundert hinein als feucht, unvollkommen und somit als schwach konzipierten kindlichen Körper. ${ }^{37}$ Die «Oekonomische Encyklopädie» von Johann Krünitz (1728-1796) vermerkt zur Manna: «Sie kann daher jedem Alter, auch den zartesten Kindern, Schwangeren und bey allen Temperamenten angewandt werden. [...] Statt aller andern Purgirmittel dient sie, wegen ihrer Süßigkeit, bey Kindern, da man sie ihnen sehr bequem, ja ohne dass sie es wissen, geben kann. ${ }^{38}$ Ein kleiner Teil der zur Anwendung gekommenen Mittel wird in den Praxisjournalen durch die Art ihrer Benutzung charakterisiert, etwa ein Pflaster oder eine Salbe. «Ob cephaleam et frigus», wegen Kopfweh und Frösteln also, griffen die Eltern des zehn Jahre alten Johann Rederlechner* zu einem nicht näher spezifizierten «Balsamum». ${ }^{39}$ Entgegen der ursprünglichen Absicht beseitigte dieser Balsam allerdings nicht etwa die eigentlichen Symptome, sondern löste vielmehr Durchfall aus. Alle diese Beschwerden schienen den Eltern noch als wenig bedrohlich. Als sich am darauffolgenden Tag jedoch ein Schmerz in der linken Seite einstellte, war für die Eltern der Punkt erreicht, an dem sie einen Arzt einschalten wollten. Die räumliche Distanz dürfte für das Zögern mit verantwortlich gewesen sein, denn immerhin lag St. Johann gute zwei Gehstunden von der Praxis entfernt, und der Fußweg zum Zeitpunkt der Er-

31 HM 509/1896, Eintrag vom 29.3.1896.

32 HM 1095/1894, Eintrag vom 6.7.1894.

33 HM 487/1896, Eintrag vom 31.3.1896.

34 HM 1041/1891, Eintrag vom 2.7.1891.

35 HM 683/1891, Eintrag vom 5.5.1891.

36 HM 1104/1896, Eintrag vom 19.8.1896.

37 Vgl. dazu Seidler 1986, 685-709, hier 690f.

38 Krünitz 1773-1858, Eintrag «Manna».

39 HM 756/1890, Eintrag vom 20.2.1890. 
krankung (Februar) war wegen Schnee, Eis und Kälte beschwerlich und mit einigen Gefahren verbunden.

Nach Durchsicht derjenigen Krankengeschichten aus den 1890er Jahren, die Hinweise auf medizinische Selbsthilfe und die Anwendung von diversen Hausmitteln enthalten, scheint der «Expertenstatus», den Ottenthal hinsichtlich der Behandlung kranker Kinder bei einem Teil der Talbevölkerung eingenommen hatte, deutlicher an Profil zu gewinnen. Nicht wenige Eltern entschlossen sich zur ärztlichen Inanspruchnahme, wenn vorerst als harmlos eingestufte Symptome wie Bauchweh, Durchfall oder Erkältungsbeschwerden nicht mit den zur Verfügung stehenden Hausmitteln zum Verschwinden gebracht werden konnten oder wenn sie über einen ungewöhnlich langen Zeitraum hinweg andauerten, so dass die Konstitution des Kindes allzu schnell geschwächt zu werden drohte. Die Entscheidung für ärztliche Hilfe wurde beschleunigt, wenn plötzlich Symptome auftauchten, die eine Abweichung vom gewohnten Krankheitsverlauf anzeigten und von den Eltern wie im Falle der Seitenschmerzen in den HM 756/1890 als Vorboten einer bedrohlichen Erkrankung (etwa einer Lungenentzündung) wahrgenommen wurden. Die dementsprechenden kindermedizinischen Therapieangebote Franz v. Ottenthals wurden von den Eltern sehr wohl in Anspruch genommen.

\section{Nachbarschaftshilfe und nicht approbierte TherapeutInnen}

Während Eltern bei einigen Erkrankungen die Erfahrung gemacht hatten, dass für deren erfolgreiche Behandlung nicht zwingend ein Arzt in Anspruch genommen werden musste und der zur Verfügung stehende «Hausarzneischatz» zur Genesung vollkommen ausreichte, führte in anderen Fällen der Weg in die Praxis über andere Heilkundige. Die «Patientinnenkarriere» der 13-jährigen Anna Treffer* aus Lappach etwa begann mit der Konsultation des örtlichen Pfarrers. Aus der Anamnese durch Ottenthal erfahren wir, dass das Mädchen wegen eines seit zehn Tagen andauernden Schmerzes in der linken Seite zu dem Kirchenmann gebracht wurde..$^{40}$ Allerdings stand auch der Pfarrer trotz seiner Kenntnisse in der Pastoralmedizin diesen spezifischen Schmerzen machtlos gegenüber. «Laxans a parocho propinatum nil juvit», notierte Franz v. Ottenthal am 23. April 1894. Seine eigene Rezeptur war indessen nur wenig erfolgreicher, denn in einer zweiten Konsultation wenige Tage später stellte er fest, dass der Schmerz immer noch vorhanden

40 Diese und die folgende Konsultation: HM 718/1894, Eintrag vom 23.4. und vom 27.4.1894. 
war und noch dazu in die rechte Leiste auszustrahlen begonnen hatte. Mit der erneuten Verschreibung einer zur Gänze abgeänderten Medikation verliert sich die Spur des Mädchens als Patientin Ottenthals. Soweit die äußerst knappen Notizen eine derartige Interpretation überhaupt zulassen, scheint Ottenthal den Pfarrer weder als Konkurrenten wahrgenommen noch als «Kurpfuscher» beim zuständigen Gemeindearzt gemeldet zu haben.

Eine Konkurrenzsituation zwischen verschiedenen AnbieterInnen kindermedizinischer Versorgung scheint mir hingegen hinsichtlich der Behandlung des eineinhalbjährigen Joseph Payr* vorzuliegen. ${ }^{41}$ Dieses Kind hatte schon seit drei Wochen mit Appetitlosigkeit und übelriechenden rötlichen Abschürfungen im Mund zu kämpfen. Erste Anlaufstelle der Eltern war mit Maria Prenn in diesem Falle die 47-jährige Hebamme des Dorfes, der bei der konkreten Behandlung spezifischer Krankheiten von Säuglingen und Kleinkindern sichtlich mehr Kompetenz zugetraut wurde als dem Privatarzt in Sand. Die schnelle Erreichbarkeit, die geringeren Kosten sowie die als nicht akut gefährdend eingeschätzten Beschwerden mögen weitere ausschlaggebende Gründe für die Wahl dieser Heilperson gewesen sein. Erst als diese Erstbehandlung nicht den gewünschten Erfolg zeigte («laxans obstetricis nil juvit»), wandten sich die Eltern an das für sie nächste Glied der Therapeutenkette. Leider umfasst die Krankengeschichte dieses jungen Patienten nur eine einmalige Konsultation, so dass wir nicht über den weiteren Verlauf der Leidensgeschichte unterrichtet sind. In den 1890er Jahren ist diese Geschichte zudem der einzige Hinweis auf eine Hebamme als medizinische Expertin des Kindesalters. Es ist jedoch davon auszugehen, dass Hebammen und andere heilkundig versierte Frauen etwa bei Erkrankungen der Mundschleimhaut, die besonders bei den jüngsten Altersgruppen durch die besonderen Ernährungsverhältnisse häufig auftraten, eine Alternative zum Arzt darstellten. ${ }^{42}$

Diese Einschätzung wird durch entsprechende Passagen in der Krankengeschichte mit der Nummer 1283 aus dem Jahr 1890 unterstützt: «2 ann. prius agyrtae et mulieres superintelligentes rachitidem invenerunt, hac hieme influenzam, manus saepe ad aures movet, saepius congestiones ad caput». ${ }^{43}$ Ottenthal mokierte sich entgegen seiner üblichen neutralen Haltung gegenüber anderen Heilkundigen doch recht deutlich über jene «Quacksalber» und «oberg'scheiten» Frauen, die der kleinen Sophia* eine Rachitis andichten wollten. An dieser Stelle tauchen sie demnach wieder auf, die «alten Kinderfrauen» aus dem Ratgeber von Adalbert Czerny, die dieser als Fluch

41 HM 998/1896, Eintrag vom 22.7.1896.

42 Loetz 1993, 233-236; Ritzmann 2008, 96 f.

43 HM 1283/1890, Eintrag vom 5.5.1890.

Gesnerus 69 (2012) 
jeden Arztes ansah, weil sie unbelehrbar seien und an alten Vorurteilen und Traditionen festhielten. Ottenthal gewährt hier einen Einblick in das dichte Netz aus sozialen Beziehungen und weitverzweigter Nachbarschaftshilfe innerhalb einer Dorfgemeinschaft, welches für ihn als Außenstehenden allein mit der Berufung auf den ärztlichen Habitus schwer zu durchdringen war. ${ }^{44}$ In diesem Fall fehlte eine klare Diagnose durch Ottenthal, die Lektüre zeitgenössischer medizinischer Schriften legt allerdings nahe, dass die wenigen in dieser Notation erwähnten Symptome (häufiges Greifen mit den Händen an den Kopf, Kongestionen im Kopf) in Lehrbüchern zur Kinderheilkunde oftmals als Anzeichen eines Hydrocephalus gesehen werden, der gemäß der damaligen Lehrmeinungen mit einer beginnenden Rachitis assoziiert wurde. ${ }^{45}$

\section{Ärzte als Konkurrenten}

Dass die Inanspruchnahme eines akademischen Arztes kein Garant für den ersehnten Heilerfolg sein musste, belegt das nächste Beispiel aus den Historiae Morborum, mit dem ich den letzten Abschnitt dieses Beitrags einleiten möchte. Dieser geht Hinweisen auf die Betreuung kranker Kinder durch Fachkollegen Ottenthals aus der Ärzteschaft nach. 1892 beispielsweise begaben sich die Eltern der achtjährigen Josepha Unterlechner* zum Gemeindearzt Dr. Alois Kortleitner, um die von Fieber begleiteten Halsschmerzen der Tochter behandeln zu lassen. ${ }^{46}$ Vermutlich hatte sich der Gesundheitszustand des Mädchens im Zuge des einwöchigen Abwägens, ob nun ärztliche Hilfe erforderlich wäre oder nicht, gravierend verschlechtert. Im Eintrag des entsprechenden Heftes ist zudem von einem «exsudatum album in pharynge» (ein weißer Belag im Rachen) die Rede. «[I]verunt ad collegam Kortleitner qui dedit gargarisma», hielt Ottenthal am 24. September 1892 in seinem Journal fest. Die Eltern gingen also zunächst zu Dr. Kortleitner, der ihnen ein Gurgelwasser für die Tochter mitgab. Das verordnete Mittel erleichterte der Patientin zwar die Atmung, verursachte

44 Weitere Hinweise auf die Inanspruchnahme anderer Heilkundiger gibt Taddei 2010,126-130. 45 So etwa im Lehrbuch von Carl Gerhardt aus dem Jahre 1861: «Wird z.B. ein Kind von zwei Monaten hydrocephalisch, so greift es nach dem Kopfe und macht vielleicht ein düsteres Gesicht. Hierin kann man eine Folge direkter Reizung der motorischen Nerven, der Empfindungscentra, oder der betreffenden sensitiven Fasern im Gehirn, oder anderseits eine gewisse Bewegung sehen, die durch Kopfschmerz hervorgerufen wurde.» Gerhardt 1861, 21.

$46 \ll 8$ ann ante septimanam adficiebatur angina febrili cum exsudato albo in pharynge iverunt ad collegam Kortleitner qui dedit gargarisma, quod excoriationes dolorificas causavit, deglutitionem fecit liberiorem - excoriationes adhuc multum dolent.» HM 1686/1892, Eintrag vom 24.9.1892. 
jedoch zugleich schmerzhafte Abschürfungen im Rachenraum. So erzählten es die Eltern jedenfalls Ottenthal, von dem sie eine Linderung der äußerst schmerzhaften «excoriationes» erwarteten. Vom Gemeindearzt, dessen verschriebenem Medikament sie offensichtlich eher die Schuld an den neuen Beschwerden gaben als der eigentlichen Krankheit, war nach Ansicht der Eltern nichts mehr zu erwarten. Dieser hatte den ihm entgegengebrachten Vertrauensvorschuss eingebüßt. Vielleicht war es Ottenthal aber auch nur gelungen, sich durch seine damals schon über 40 Jahre bestehende Praxis als vertrauensvoller «Hausarzt» der Familie zu positionieren, oder vielleicht erfüllte er mit der Verschreibung der Belladonna und des Chinin eher die Erwartungen der Eltern an ein vertrautes Medikament. Beides hätte ihm einen erheblichen Konkurrenzvorteil gegenüber dem erst seit wenigen Jahren in der Gemeinde tätigen und beinahe zwei Generationen jüngeren Kortleitner verschafft. Schließlich erhielt der 1861 in Brixen geborene Kortleitner 1888 unmittelbar nach Beendigung seines in Graz, Wien und Innsbruck absolvierten Medizinstudiums die Stelle als Gemeindearzt des Sanitätssprengels Sand-Taufers und musste sich erst bewähren. ${ }^{47}$

Enttäuscht von Kortleitner zeigten sich jedenfalls auch die Eltern eines sechsjährigen Kindes aus Ahornach, als sie sich von ihm eine Abhilfe des «ex partu», also seit der Geburt bestehenden Augenleidens erhofften. ${ }^{48}$ Nachdem das «Übel» trotz der Medikation Kortleitners erneut dermaßen stark aufgetreten war, dass dem Knaben die Augen zugeschwollen waren, fanden sich die ratlosen Eltern am 22. Dezember 1891 schlussendlich in Ottenthals Praxis ein. «[M]edic. Collegae Kortleitn nil juvit», kommentierte Ottenthal mit knappen Worten den glücklosen Heilversuch des jüngeren Kollegen. ${ }^{49}$ Mit dem Spitalsarzt von Bruneck, Dr. Georg Wagner, und dem Stadtarzt von Bruneck, Dr. Jakob Erlacher, tauchen $1896^{50}$ zwei weitere Ärzte aus dem näheren Einzugsgebiet Ottenthals in seinen Krankengeschichten auf, von deren fehlgeschlagenen bzw. nicht den gewünschten Erfolg bringenden Erstbehandlungen der Privatarzt profitierte.

Andererseits musste zwischen den einzelnen Ärzten einer Region nicht zwingend ein Konkurrenzverhältnis bestehen. Die durch Sanitätsgesetze genauestens geregelten Funktionen eines fix besoldeten Gemeindearztes, eines angestellten Spitalarztes oder eines auf Privatkunden angewiesenen

47 TLA, Akten Statthalterei 1896, Sanität; Zl. 37885/2892, liegend in ZL. 37621/2870 ad 1015. «Bericht der kk. Bezirkshauptmannschaft Bruneck über den Abgang des Dr. Alois Kortleitner vom Land.»

48 HM 1780/1891, Eintrag vom 22.12.1891.

49 HM 1780/1891, Eintrag vom 22.12.1891.

$50 \ll \ldots$ ante 4 dies post ordinationem Dr Wagner hinc inde delirat ...». HM 1127/1896, Eintrag vom 27.8.1896. 
praktischen Arztes führte vor allem gegen Ende des 19. Jahrhunderts zu entsprechenden Zuständigkeitsbereichen und zu Spezialisierungen bei den angebotenen medizinischen Leistungen, die jeweils unterschiedliche PatientInnenschichten ansprachen. Nicht zuletzt war gerade in einem Gebiet wie dem Tauferer Ahrntal mit seiner geringen Ärztedichte eine enge Zusammenarbeit zwischen den wenigen Kollegen aus der Ärzteschaft häufig erforderlich. Die genaue Ursache etwa der seit zwei Wochen bestehenden, vagen Gliederschmerzen eines zehnjährigen Jungen stellten Ottenthal vor ein dermaßen großes Rätsel, dass er sich mit Dr. Kortleitner über diesen Fall austauschte. Als Gemeindearzt kannte dieser die Leidensgeschichte des Knaben offensichtlich aus eigener Anschauung. «Kortleitner vidit vitium cordis», notierte Ottenthal folglich anlässlich der nächsten Konsultation des Jungen rund einen Monat nach der ersten Behandlung, auch wenn er selbst eher der Diagnose einer rheumatischen Fiebererkrankung zugeneigt schien. $^{51}$

Gegenseitige Überweisungen waren in diesem Sprengel ebenfalls keine Seltenheit, etwa im Falle von Elisabeth Rautter* aus Sand, deren Diphtherieerkrankung zunächst eine Woche lang von Kortleitner behandelt wurde. Als diese aber «in decremento» begriffen war und keine akute Lebensgefahr mehr zu bestehen schien, übernahm Ottenthal die weitere Behandlung. ${ }^{52}$ Vermutlich hingen derartige Patientenüberweisungen mit den sanitätspolitischen Regelungen für ansteckende Krankheiten zusammen, laut denen der von der Sanitätsbehörde im Falle einer Seuche bestellte «Epidemiearzt» nach einer ersten Versorgung und nach Abklingen der größten Lebensgefahr zur weiteren Behandlung an einen anderen Arzt überweisen konnte, um die Kosten für den Staat gering zu halten. ${ }^{53} \mathrm{Im}$ Falle ausgedehnter Epidemien unterstützten diese von den Gemeinden angeforderten «Vertretungsärzte» auch den eigentlichen Gemeindearzt bei seiner Arbeit. ${ }^{54}$ Für Ottenthal sollte sich die weiterführende Versorgung des kranken Mädchens als fatal erweisen, denn das Mädchen verstarb schließlich zwei Wochen später an dieser heimtückischen Krankheit, die in den frühen 1890er Jahren in mehreren Epidemiewellen noch viele weitere Opfer hauptsächlich unter der jungen Talbevölkerung fordern sollte.

51 HM 227/1893, Eintrag vom 7.2.1893 sowie die weiteren Konsultationen in diesem und den folgenden Jahren.

52 «continuet med Dr. Kortleitn.» HM 933/1893, Eintrag vom 5.6.1893.

53 Als Beispiel für diese geübte Praxis vgl. die Schritte der Medizinalverwaltung zur Eindämmung einer im Jahre 1860 grassierenden Typhusepidemie im Ahrntal. Siehe Unterkircher 2008, 202-208.

54 Vgl. Taddei 2008, 231f. 


\section{Schlussbetrachtung}

Kinder (1.-14. Lebensjahr) stellten in den für diesen Beitrag untersuchten Krankenjournalen der 1890er Jahre zwar nicht die anteilsmäßig größte, aber dennoch eine wichtige Gruppe der Patienten Franz v. Ottenthals dar. Als praktischer Arzt konnte er sich demnach mit einigem Erfolg auf dem medizinischen Markt für Kinderkrankheiten behaupten. Der hier gewählte Fokus auf Kinder als spezifische Patientengruppe eines Arztes machte folglich deutlich, dass Eltern bei einigen alterstypischen Beschwerden (etwa bei Diphtherie) einem akademischen Arzt sehr viel Vertrauen entgegenbrachten und mitunter weite Wege in Kauf nahmen, um Medikamente für ihren erkrankten Nachwuchs verschrieben zu bekommen. Entgegen den zeitgenössischen Klagen der Ärzte über eine mangelnde Inanspruchnahme medizinischer Hilfe bei (Klein-)Kindern aus einer angeblichen Sorglosigkeit heraus bestand sehr wohl eine Nachfrage nach der Behandlung erkrankter Minderjähriger durch akademische Ärzte. Wie die entsprechenden Fallgeschichten in den Historiae Morborum zu den jungen Patientinnen und Patienten deutlich belegen, überließen Eltern ihre Kinder im Erkrankungsfall weder fatalistisch dem Willen Gottes, noch suchten sie ausnahmslos Hilfe bei LaienheilerInnen. Lediglich ein geringer Teil der die Altersgruppe der Kinder betreffenden Krankengeschichten enthält Hinweise auf medizinische Selbsthilfe oder auf TherapeutInnen, die ebenfalls kinderheilkundliche Dienstleistungen anboten. Zudem ergab sich das Bild, dass eine mögliche Konkurrenz am kinderheilkundlichen Markt nicht nur von «KurpfuscherInnen» oder Hebammen ausging, sondern auch von Ärzten aus der näheren Umgebung. In den Historiae Morborum sind Vorbehandlungen durch Ärztekollegen zwar nicht häufig vermerkt worden. Doch selbst die wenigen Hinweise legten nahe, dass Franz v. Ottenthal seine medizinischen Angebote für die Behandlung kranker Kinder sehr gut «anpreisen» musste, um die Nachfrage besorgter Eltern nach seinen spezifischen Kenntnissen wachzuhalten. «Nicht für jedes Leiden galten dieselben Heilkundigen als kompetent.» ${ }^{55}$ Diese aus den Quellen des 18. Jahrhunderts gewonnene Erkenntnis von Iris Ritzmann bleibt mit gewissen Einschränkungen auch noch gut 100 Jahre später für Ottenthal gültig - allen im 19. Jahrhundert stattfindenden Medikalisierungs- und Professionalisierungsschritten des Medizinalwesens zum Trotz.

55 Ritzmann 2003, 173. 


\section{Archivsiglen}

SLA Südtiroler Landesarchiv

TLA Tiroler Landesarchiv

\section{Bibliographie}

Czerny, Adalbert, Der Arzt als Erzieher des Kindes (Leipzig/Wien $\left.{ }^{8} 1934\right)$

Das österreichische Sanitätswesen, Beiblatt vom 29. Jänner 1891 und vom 30. April 1891

Dietrich-Daum, Elisabeth, «Die Klage der Ärzte. Marktprobleme und Professionalisierung in der zweiten Hälfte des 19. Jahrhunderts in Österreich», bricolage. Innsbrucker Zeitschrift für Europäische Ethnologie 5: medikale Kulturen (2008) 210-221

Dinges, Martin, «Immer schon 60\% Frauen in den Arztpraxen? Zur geschlechterspezifischen Inanspruchnahme des medizinischen Angebots (1600-2000)», in: Martin Dinges (Hrsg.), Männlichkeit und Gesundheit im historischen Wandel ca. 1800-ca. 2000 (Stuttgart 2007) 295-322

Egger, Gertrud, «Von Badern, Barbieren und Wundärzten. Medizin und Heilkunst im Ahrntal», in: Gemeinde Ahrntal (Hrsg.), Ahrntal. Ein Gemeindebuch (Steinhaus 1999) 60-69

Gerhardt, Carl, Lehrbuch der Kinderkrankheiten (Tübingen 1861)

Innerhofer, Josef, Taufers Ahrn Prettau. Die Geschichte eines Tales (Bozen 1980)

K.K. Statistische Central-Commission (Hrsg.), Special-Orts-Repertorium von Tirol. Neubearbeitung auf Grund der Ergebnisse der Volkszählung vom 31. December 1890 (Wien 1893)

K.K. Statistische Central-Commission (Hrsg.), Statistik des Sanitätswesens der im Reichsrathe vertretenen Königreiche und Länder für das Jahr 1879 (Wien 1882)

Krünitz, Johann Georg, Oekonomische Encyklopädie oder allgemeines System der Staats-, StadtHaus- und Landwirthschaft, 242 Bände (Brünn 1773-1858)

Loetz, Francisca, Vom Kranken zum Patienten. 〈Medikalisierung〉 und medizinische Vergesellschaftung am Beispiel Badens 1750-1850 (Stuttgart 1993)

Messner, Wolfgang, Wirtschafts- und Sozialgeschichte des Tauferer Tales/Ahrntales im ausgehenden 18. und im Laufe des 19. Jahrhunderts (unveröffentl. Dipl. phil. Innsbruck 2004)

N.N., «Fortschritte in der Organisation des Gemeinde-Sanitätsdienstes», Das österreichische Sanitätswesen 9 (1897), 482-485

Pammer, Michael, «Die Diphtherie in den im Reichsrat vertretenen Königreichen und Ländern in den Jahren 1880 bis 1912», in: Elisabeth Dietrich-Daum/Roberto Taiani (Hrsg.), Medikalisierung auf dem Lande/Medicalizzazione in area alpina. Geschichte und Region/Storia e Regione 14 (2005) 70-89

Dr. W. Papes Griechisch-Deutsches Handwörterbuch. In drei Bänden, erster Band: A-K (Braunschweig $\left.{ }^{3} 1908\right)$

Ritzmann, Iris, «Die jüngsten Patienten Hahnemanns - eine analytische Studie zur Kinderpraxis in den Anfängen der Homöopathie», Medizin, Gesellschaft und Geschichte 18 (1999) 189-208

Ritzmann, Iris, «Der Faktor Nachfrage bei der Ausformung des modernen Medizinalwesens Überlegungen am Beispiel der Kinderheilkunde», in: Bettina Wahrig/Werner Sohn (Hrsg.), Zwischen Aufklärung, Policey und Verwaltung. Zur Genese des Medizinalwesens 1750-1850 (Wiesbaden 2003) 163-178

Ritzmann, Iris, Sorgenkinder. Kranke und behinderte Mädchen und Jungen im 18. Jahrhundert (Köln/Weimar/Wien 2008)

Ruisinger, Marion Maria, «Die Kinder sind überhaupt schwerer zu curiren als Erwachsene». Junge Patienten in der Konsiliarkorrespondenz des frühen 18. Jahrhunderts», Gesnerus 62 (2005) 218-236

Seidler, Eduard, «Das kranke Kind. Historische Modelle einer medizinischen Anthropologie des Kindesalters», in: Jochen Martin/August Nitschke (Hrsg.), Zur Sozialgeschichte der Kindheit (Freiburg/München 1986) 685-709

Stolberg, Michael, «Patientenschaft und Krankheitsspektrum in ländlichen Arztpraxen des 19. Jahrhunderts», Medizinhistorisches Journal 28 (1993) 3-27 
Taddei, Elena, «Bestellungsverfahren von Ärzten in Tirol in der zweiten Hälfte des 19. Jahrhunderts: Qualifikationen, Auswahlkriterien und Erwartungen», in: Dietrich-Daum, Elisabeth u.a.(Hrsg.), Arztpraxen im Vergleich:18.-20. Jahrhundert (Innsbruck/Wien/Bozen 2008) 221-237

Taddei, Elena, Franz von Ottenthal. Arzt und Tiroler Landtagsabgeordneter (1818-1899) (Wien/Köln/Weimar 2010)

Unterkircher, Alois, «Ein Südtiroler Landarzt, seine Praxis und ein typhöser Seuchenzug, oder:Warum Männer, die 1860 ihre Wäsche nicht selbst wuschen, mitunter länger lebten als Frauen», in: Dietrich-Daum, Elisabeth u.a. (Hrsg.), Arztpraxen im Vergleich: 18.-20. Jahrhundert (Innsbruck/Wien/Bozen 2008) 193-217

Wolff, Eberhard, «Der «willkommene Würgeengel〉. Verstehende Innenperspektive und 〈genaue〉 Quelleninterpretation - am Beispiel der erwünschten Kindertode in den Anfängen der Pockenschutzimpfung», in: Martin Dinges/Thomas Schlich (Hrsg.), Neue Wege in der Seuchengeschichte (Stuttgart 1995) 105-141 